\title{
Curcumin and Freshwater Clam Extracts Alleviate the Progression of Osteoarthritis by Reducing Synovial Inflammation and Allowing Cartilage Regeneration
}

\author{
Chiao-Hsu Ke ${ }^{1}$, Chia-Hui Hsu ${ }^{1}$, Yu-Ling Lin ${ }^{2}$ D, Wei-Hsiang Huang ${ }^{3}$, Hsin-Pei Weng ${ }^{1}$, Yi-Tzu Ke ${ }^{1}$ \\ and Chen-Si Lin ${ }^{1, *(D)}$ \\ 1 Department of Veterinary Medicine, School of Veterinary Medicine, National Taiwan University, \\ Taipei 10617, Taiwan; f08629002@ntu.edu.tw (C.-H.K.); jesperhsu.dvm@gmail.com (C.-H.H.); \\ r07629003@ntu.edu.tw (H.-P.W.); eva200064@gmail.com (Y.-T.K.) \\ 2 Agricultural Biotechnology Research Center, Academia Sinica, Taipei 11529, Taiwan; \\ lyring@gate.sinica.edu.tw \\ 3 Graduate Institute of Molecular and Comparative Pathobiology, School of Veterinary Medicine, National \\ Taiwan University, Taipei 10617, Taiwan; whhuang@ntu.edu.tw \\ * Correspondence: cslin100@ntu.edu.tw; Tel.: +886-233-661-286
}

check for updates

Citation: Ke, C.-H.; Hsu, C.-H.; Lin, Y.-L.; Huang, W.-H.; Weng, H.-P.; Ke, Y.-T.; Lin, C.-S. Curcumin and Freshwater Clam Extracts Alleviate the Progression of Osteoarthritis by Reducing Synovial Inflammation and Allowing Cartilage Regeneration. Processes 2021, 9, 931. https:// doi.org/10.3390/pr9060931

Academic Editor: Chao-Min Wang

Received: 13 March 2021

Accepted: 24 May 2021

Published: 25 May 2021

Publisher's Note: MDPI stays neutral with regard to jurisdictional claims in published maps and institutional affiliations.

Copyright: (c) 2021 by the authors. Licensee MDPI, Basel, Switzerland. This article is an open access article distributed under the terms and conditions of the Creative Commons Attribution (CC BY) license (https:/ / creativecommons.org/licenses/by/ $4.0 /)$.

\begin{abstract}
Osteoarthritis (OA) is a common degenerative disorder and is accompanied by numerous pain symptoms. With increased age, individuals develop a chronic inflammatory status, and pro-inflammatory cytokines as well as mediators contribute to the progression of OA. However, no desirable remedies have been completely able to inhibit OA progression or safely provide effective symptomatic relief. Natural component extracts or dietary-derived compounds are widely used for anti-inflammatory diseases. Curcumin and freshwater clam extract (FCE) have been proven as functional foods that are able to regulate immune systems. This study demonstrated that curcumin and FCE had synergistic effects on alleviating the progression of OA by assuaging inflammation and repairing the cartilage within the joints. After consumption of curcumin and FCE, the severity of synovitis was quantified by the infrapatellar fat pad inflammation scoring system and the Osteoarthritis Research Society International (OARSI) scoring system. Significant improvement and articular cartilage regeneration were noted. Moreover, once the inflammation within the joints was reduced, the animals redistributed their body weight on the OA-induced hindlimb. In summary, curcumin and FCE possess desirable anti-inflammatory and repair functions, suggesting their potential as alternative remedies in the management of OA or other inflammatory diseases.
\end{abstract}

Keywords: osteoarthritis; curcumin; freshwater clam extracts; cartilage regeneration; anti-inflammation

\section{Introduction}

Osteoarthritis (OA) is a complex disease characterized by loss of articular cartilage and failure of the synovial joint [1,2]. Clinical signs of OA include joint swelling, joint pain, limitation of activity, stiffness, and immobility. The chronic and progressive illness ultimately leads to irreversible destruction of articular cartilage and inflammation of the infrapatellar fat pad (IFP) [3]. Even though OA is one of the most common degenerative disorders in ageing society, the exact etiology or pathogenesis has not been well-defined [4,5]. To date, no desirable remedies have been completely able to inhibit OA progression or provide effective symptomatic relief $[1,2,4]$.

Surgical intervention, pain relievers, and complementary medicines are now mainstream medicines for OA patients. Surgical intervention includes partial or total joint replacement (TJR), which can immediately improve severe clinical symptoms [2,6]. Nevertheless, it is rather aggressive and costly [7]. Pain relievers mainly target pain. Specifically, non-steroidal anti-inflammatory drugs (NSAIDs) are usually considered the first-line medical treatment against OA [2,8]. However, there is some question about their safety. Namely, 
prolonged use of NSAIDs correlates with several side effects, such as cardiovascular risk and gastro-intestinal toxicity $[5,9,10]$. Furthermore, although they reduce pain, NSAIDs are unable to slow the progression or counteract the pathophysiology of OA [11]. Consequently, non-drug approaches for OA treatment regimens are increasingly needed.

Many dietary-derived compounds are considered to have effective anti-inflammatory and protective functions, two examples being curcumin and freshwater clams [11-13]. Curcumin is a yellowish pigment extracted from the rhizome of turmeric (Curcuma longa) [14]. It is also a natural antioxidant with low toxicity and possesses not only anti-inflammatory but anti-tumor properties $[14,15]$. Pharmacological evidence suggests that curcumin is a therapeutic agent or immune modulator in inflammatory diseases such as pancreatitis, rheumatoid arthritis, and inflammatory bowel diseases [16,17]. Curcumin modulates the inflammatory response by downregulating the activity of cyclooxygenase-2 (COX-2) [18] and inhibits the production of pro-inflammatory cytokines by targeting the nuclear factor kappa-light-chain-enhancer of the activated B cell (NF-kB) pathway [19]. Likewise, the potent anti-inflammatory properties of freshwater clams (Corbicula fluminea) have also been reported [20]. Freshwater clam extract (FCE) has been widely studied as a potential immune regulator that attenuates inflammatory responses [21-23]. FCE has demonstrated a hepatoprotective function $[21,22]$ and inhibitory effects on pro-inflammatory cytokine release, such as TNF- $\alpha$ and IL-1 $\beta$, as found by in vivo and in vitro studies [23].

Considering the synergistic role of herbal supplements and nutrient extracts in the repair or reduction of OA progression, this study utilized an induced-OA animal model to assess the clinical manifestations under continuous administration of curcumin and FCE. In addition, to investigate the effects of curcumin and FCE, this study validated the anti-inflammatory effects within the synovium and the cartilage repair abilities of these combined components.

\section{Materials and Methods}

\subsection{MIA-Induced OA Animal Model for Cartilage Regeneration and Synovial Repair Evaluation}

Male Sprague-Dawley rats, $350.67 \pm 9.85 \mathrm{~g}$ in weight, were purchased from the National Science Council Animal Center, Taipei, Taiwan. All rats were maintained in the Department of Veterinary Medicine, School of Veterinary Medicine, National Taiwan University, Taipei, Taiwan, and kept under a 12/12 h light/dark cycle with food and water available ad libitum. The rats were anesthetized under $1.5 \%$ isoflurane inhalation, and then $2.5 \mathrm{mg}$ of monosodium iodoacetate (MIA) (Sigma, St. Louis, MO, USA) dissolved in $50 \mu \mathrm{L}$ sterile $0.9 \%$ saline was administered into the left knee articular cavity of rats by a 31-gauge needle. At the same time, $50 \mu \mathrm{L}$ saline solution was injected into the right knee as a control. After the injected hindlimb was stretched and flexed five times, the rats were returned to their cages. On day 0 , the rats were randomly distributed into one of three groups: high-dose curcumin and FCE p.o. (HCF), low-dose curcumin and FCE p.o. (LCF), and vehicle control ( $n=9$ to 12 per group). The HCF and LCF groups were orally administered 600 and $400 \mathrm{mg}$ curcumin and FCE per diem respectively, while vehicle control rats were fed $100 \%$ corn oil per diem in a total volume of $1 \mathrm{~mL}$. Weight-bearing status (WBS) tests were performed on day 0 (baseline, the day before MIA injection) and on different days until the end of this experiment. Then, the rats were sacrificed, and bilateral knee joints were collected for pathological studies. The experimental design is shown in Figure 1a. In vivo experimental protocols were approved by the Institutional Animal Care and Use Committee, National Taiwan University, Taipei, Taiwan (IACUC No. NTU107-EL-00189).

\subsection{Measuring Hindlimb Weight-Bearing Distribution}

To evaluate the change in weight-bearing capability, hindlimb WBS tests were measured using an incapacitance tester (Linton Instrumentation, Norfolk, UK). Weight distribution was measured between left treated (intra-articular injection of $2.5 \mathrm{mg}$ MIA) and normal right hindlimbs. Rats were carefully placed in a measuring chamber such that each hindlimb rested on a separate force plate. The weight-bearing force exerted by 
each hindlimb was averaged over $10 \mathrm{~s}$. Each datum is the mean of five readings. The weight distribution ratio onto the treated hindlimb was calculated as (weight on the left hindlimb/weight on the bilateral hindlimb) $\times 100 \%$ [24].

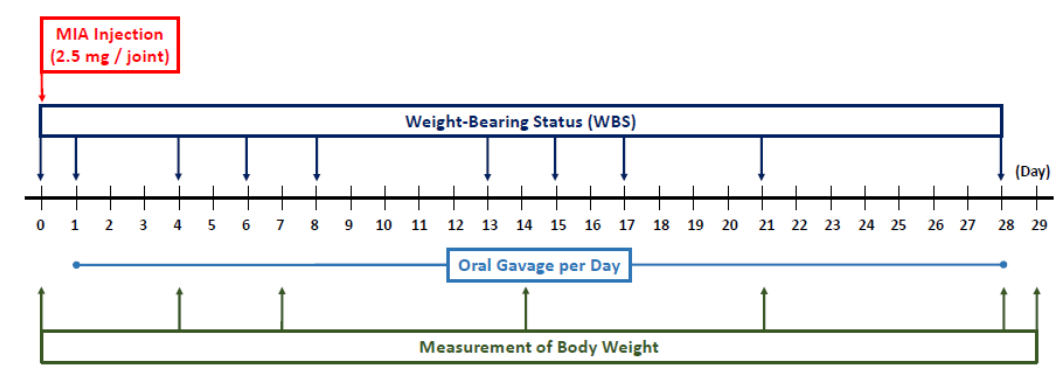

(a)

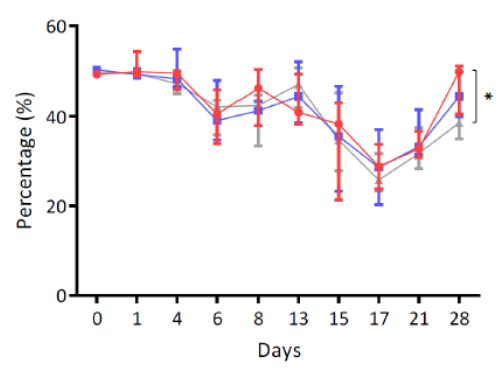

(b)

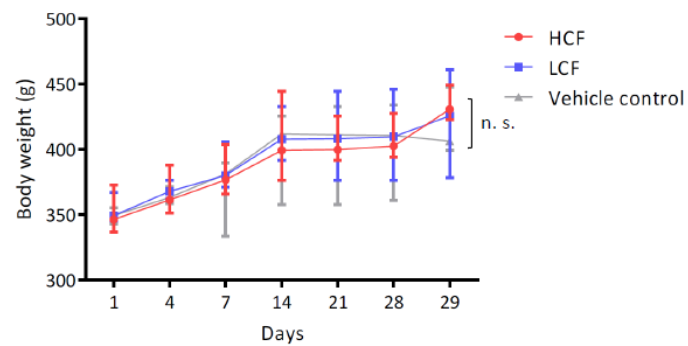

(c)

Figure 1. Experimental schema, weight-bearing status (WBS) test results, and body weight change of MIA-induced osteoarthritis rat model. (a) Sprague-Dawley rats were injected with $2.5 \mathrm{mg}$ monoiodoacetic acid (MIA) dissolved in $50 \mu \mathrm{L}$ sterile $0.9 \%$ saline per joint to induce osteoarthritis (OA) at the beginning of the experiment. Saline only was administrated into the right knee articular cavity of rats as a control. Oral gavage of curcumin and freshwater clam extract (FCE) or 100\% corn oil for the control group was executed every single day. Body weight and weight-bearing status (WBS) were measured as presented above. (b) WBS test was performed on days 0 (baseline), 1, 4, 6, 8, 13, 15, 17, 21, and 28. WBS test results of the left hindlimb on day 28 showed a significant difference between vehicle control and HCF group $(p<0.05)$. (c) Body weights of all groups had no significant differences at every measurement timepoint with an upward tendency. All data are presented as mean \pm S.D. WBS test and body weight were analyzed with two-way ANOVA, followed by Tukey's post hoc test (HCF, high-dose curcumin and FCE p.o.; LCF, low-dose curcumin and FCE p.o.; n.s., no significant difference; $\left.{ }^{*}, p<0.05\right)$.

\subsection{Histopathological Examination for Synovial Inflammation and Cartilage Regeneration}

Knee joints were fixed in $10 \%$ neutral formalin for 7 days, decalcified in rapid decalcifying solution (Sigma, St. Louis, MO, USA) for 5 days, and then embedded in paraffin wax after being harvested. Deparaffinized slides were sagittally sectioned at $4 \mu \mathrm{m}$ and stained with Hematoxylin and Eosin (H\&E) and with Safranin O/Fast green stain. For the assessment of synovitis after MIA induction, an infrapatellar fat pad (IFP) inflammation scoring system on a scale of 0 to 6 points was used (Table 1) [3,25]. To evaluate the cartilage degeneration, damage, and regeneration, the Osteoarthritis Research Society International (OARSI) score was employed on a scale of 0 to 24 points [26].

\subsection{Immunohistochemical Staining and Scoring System}

The sections which have been prepared as previously described underwent antigen retrieval in boiling citrate buffer $(\mathrm{pH}=6.0)$ for $20 \mathrm{~min}$. To block non-specific endogenous binding, sections were treated with hydrogen peroxide for $60 \mathrm{~min}$; then, the slides were incubated with primary antibody (IL-1 $\beta$, COX-2, and TNF- $\alpha$; 1:200 dilution) at room temperature overnight, followed by HRP-conjugated secondary antibody. The expression levels were detected using the TAlink mouse/rabbit polymer detection system (TAHC04D, BioTnA Biotech, Kaohsiung, Taiwan). A semi-quantitative method was used to score cytokine expression in all tissue sections, as previously described [27]. Briefly, Figure 2 shows the cytokines 
expressed in sections that were analyzed regarding staining intensity using a score from 0 to 3 $(0=$ no staining, 1 = weak staining, $2=$ moderate staining, $3=$ strong staining $)$. The staining intensity was counted in 5 random microscopic fields at $40 \times$ objective magnification in each sample independently, and separately scored by two board-certified veterinary pathologists from NTU veterinary hospital, blinded to the experimental history.

Table 1. Synovitis score and fibrosis score using the infrapatellar fat pad (IFP) inflammation scoring system (0-6).

\begin{tabular}{cc}
\hline Points & Histological Features \\
\hline 0 & Cell infiltration at the surface of IFP \\
Normal \\
Cellularity is increased, multinucleated cells present \\
2 & Thickened lining cells, low (<three-fold thickness of the normal synovium $)$ \\
3 & Thickened lining cells, high (>three-fold thickness of the normal synovium) \\
\hline Points & Histological Features \\
\hline & Fibrosis in the body of the IFP \\
1 & No fibrotic lesion \\
2 & Fibrotic lesion in infrapatellar fat pad present, low \\
3 & Fibrotic lesion increased, high \\
\hline
\end{tabular}

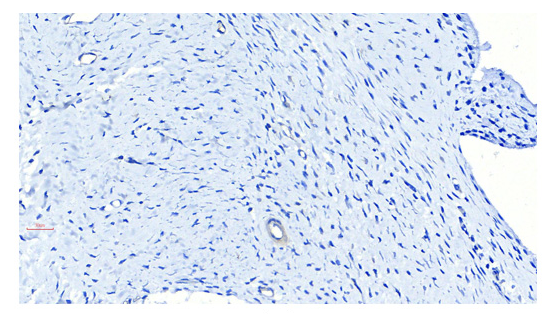

(a)

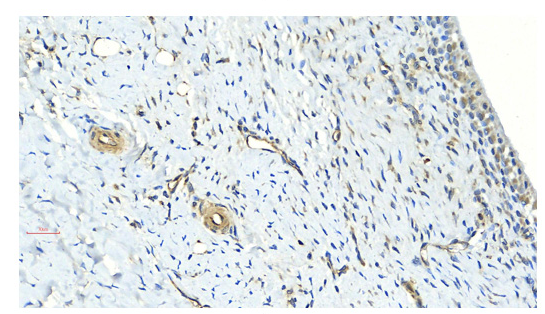

(c)

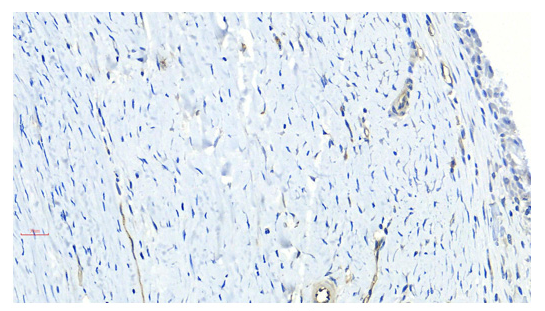

(b)

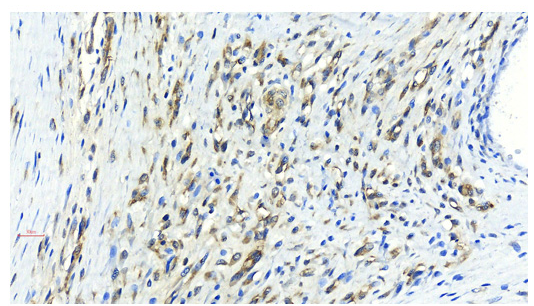

(d)

Figure 2. Scoring system from 0 to 3 for immunohistochemical staining intensity. Four representative histopathological features with different intensities for staining grades with cytokine expression are shown. Scale bar represents $30 \mu \mathrm{m}$. (a) Intensity score 0, (b) Intensity score 1, (c) Intensity score 2, (d) Intensity score 3.

\subsection{ELISA}

Blood was collected from experimental rats on day 21 and sera was obtained via centrifugation $(6000 \times g$ for $15 \mathrm{~min})$ at $4{ }^{\circ} \mathrm{C}$. MCP-1 (Biolegend, San Diego, CA, USA), Interleukin (IL)-1 $\beta$, IL-6, and TNF- $\alpha$ concentrations in the serum were evaluated using ELISA kits (R\&D system, Minneapolis, MN, USA), following the manufacturer's protocol.

\subsection{Statistical Analysis}

Data are presented as mean \pm standard deviation (S.D.) in this study. Using GraphPad Prism 7.0. software, the WBS tests and body weights were analyzed with two-way ANOVA, followed by Tukey's post hoc test. Kruskal-Wallis test followed by post hoc Dunn's tests was used for scoring analysis. $p$-values less than 0.05 were considered to indicate a significant difference. 


\section{Results}

\subsection{High-Dosage Curcumin and FCE Can Effectively Relieve Clinical Symptoms Safely}

The main clinical signs of OA are chronic joint pain and limitation of activity. Based on the characteristics of the MIA-induced OA model, measuring hindlimb weight-bearing percentage was considered a universal and practical method to evaluate the development of OA. The WBS test is able to detect joint injury and recovery status from changes in weight distribution between affected and controlled hindlimbs [24]. Intra-patellar injection of MIA led to a painful response in the ipsilateral treated hindlimb, causing the animals to redistribute their body weight to the contralateral limb. For the WBS test, $2.5 \mathrm{mg}$ MIA injection induced a remarkable reduction in ipsilateral weight-bearing on day 6 in all groups (Table 2). These clinical findings confirmed that our MIA-induced OA rat model presented successfully.

Table 2. Results of the weight-bearing status test (percentage, $\%$ ). WBS test was performed on day 0 (baseline) and different days until the end of this experiment. All data were presented as mean \pm S.D. and analyzed with two-way ANOVA, followed by Tukey's post hoc test (HCF, high-dose curcumin and FCE p.o.; LCF, low-dose curcumin and FCE p.o.; * $p<0.05)$.

\begin{tabular}{cccc}
\hline Days & Vehicle Control Group & HCF Group & LCF Group \\
\hline 0 (Baseline) & $49.75 \pm 0.45$ & $49.38 \pm 0.50$ & $50.38 \pm 0.18$ \\
1 (MIA injection) & $49.50 \pm 0.54$ & $50.88 \pm 2.15$ & $49.50 \pm 0.93$ \\
4 & $47.50 \pm 1.90$ & $48.95 \pm 1.74$ & $49.63 \pm 3.18$ \\
6 & $40.95 \pm 3.02$ & $40.23 \pm 5.51$ & $40.25 \pm 5.17$ \\
8 & $40.78 \pm 4.37$ & $45.25 \pm 4.88$ & $40.98 \pm 1.99$ \\
13 & $46.83 \pm 3.17$ & $42.40 \pm 4.37$ & $44.93 \pm 4.95$ \\
15 & $35.65 \pm 6.22$ & $35.28 \pm 8.81$ & $35.33 \pm 8.35$ \\
17 & $26.68 \pm 3.32$ & $28.83 \pm 3.54$ & $28.68 \pm 5.96$ \\
21 & $32.38 \pm 3.32$ & $33.28 \pm 2.20$ & $34.88 \pm 3.96$ \\
28 & $39.21 \pm 3.59 *$ & $47.96 \pm 4.33 *$ & $43.54 \pm 2.08$ \\
\hline
\end{tabular}

Compared with the vehicle control group $(39.21 \% \pm 3.59 \%)$, the HCF group $(47.96 \% \pm 4.33 \%)$ exhibited a noticeably higher weight distribution on the MIA-induced joint until the end of the observation period $(p<0.05)$. On the other hand, the LCF group $(43.54 \% \pm 2.08 \%)$ showed a slight but not significant increase in weight-bearing capacity until day 28 (Figure 1b). Furthermore, the body weight of the experimental rats was recorded regularly during the study, and there were no significant differences among all groups ( $p>0.05$, Figure 1c). As a whole, these results indicated that HCF might safely alleviate the clinical symptoms of OA.

\subsection{Curcumin and FCE Simultaneously Alleviated Local and Systemic Inflammatory Responses}

To evaluate the status of synovitis, the IFP of MIA-induced OA rats was histologically assessed after a knee joint fixation process. In the vehicle control (VC) and the LCF groups, the thickness of lining cells at the surface of the IFP highly increased. Meanwhile, fibrotic lesions were observed and occupied the majority of the IFP (Figure 3a). In short, these pathological findings concurred with previous findings that MIA-induced OA rats simulated the natural progression of OA disease in vivo. The IFP inflammation scoring system was used to assess cell infiltration at the surface of the IFP and fibrosis in the body of the IFP. The inflammation scores were significantly higher in the VC and LCF groups than in the HCF group $(p<0.01$ ) (Figure $3 b$ ). Inflammatory cytokines which play vital roles in OA progressions, such as COX-2, TNF- $\alpha$, and IL-1 $\beta$, are also analyzed by IHC within the IFP. The strong staining signal of COX-2, TNF- $\alpha$, and IL- $1 \beta$ can be observed in the VC group, indicating a progressive inflammatory response in the OA joints. In contrast, there is a significantly reduced inflammation after the curcumin and FCE consumption (Figure $4 a, b)$. Interestingly, low- and high-dosage intake of curcumin and FCE can reduce COX-2 ( $p<0.01)$; however, only HCF can effectively suppress TNF- $\alpha$ (HCF, $p<0.01$; LCF, 
$p>0.05$ ) and IL-1 $\beta$ (HCF, $p<0.01 ; \mathrm{LCF}, p>0.05$ ) expression. These results are consistent with the WBS test, and both indicate that, in the rats that consumed high doses of curcumin and FCE orally, the inflammatory response can be successfully reduced in the IFP and the microenvironment within the joint. With the decreased inflammatory responses, the curcumin and FCE lessen the pain in rats, leading to improved clinical symptoms.
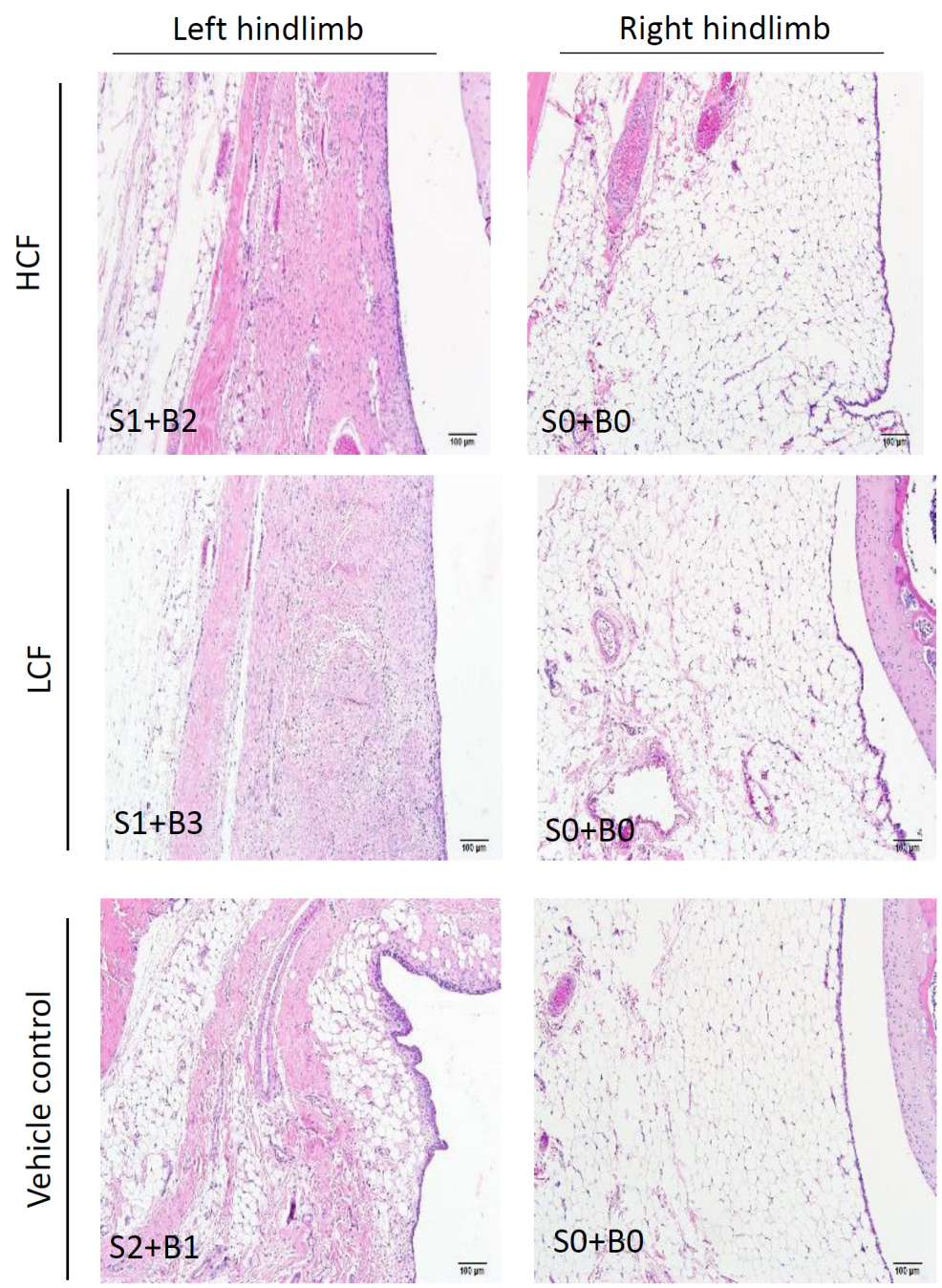

(a)

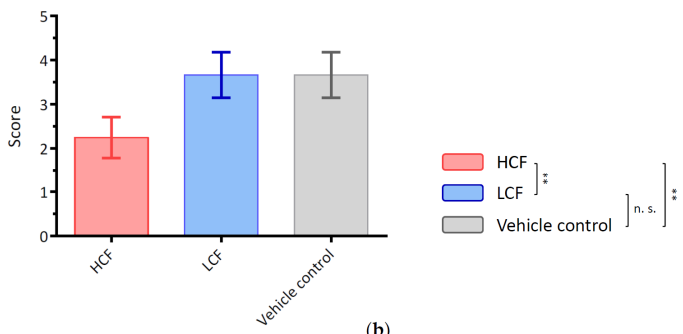

(b)

Figure 3. Representative histopathological features of synovium and infrapatellar fat pad (IFP) inflammation scores (surface and body score) using the IFP inflammation scoring system. (a) Joints were sectioned in the sagittal plane and stained with Hematoxylin and Eosin (H\&E), and representative IFP inflammation scores are shown in each image. (b) The IFP inflammation score was used to assess the synovium and the IFP. Data are presented as mean \pm S.D. and were analyzed with the Kruskal-Wallis test, followed by Dunn's post hoc test (HCF, high-dose curcumin and FCE p.o.; LCF, low-dose curcumin and FCE p.o.; L, the left hindlimb; R, the right hindlimb; S, the surface of the IFP; $\mathrm{B}$, the body of the IFP; n.s., no significant difference; ${ }^{* *}, p<0.01$ ). 

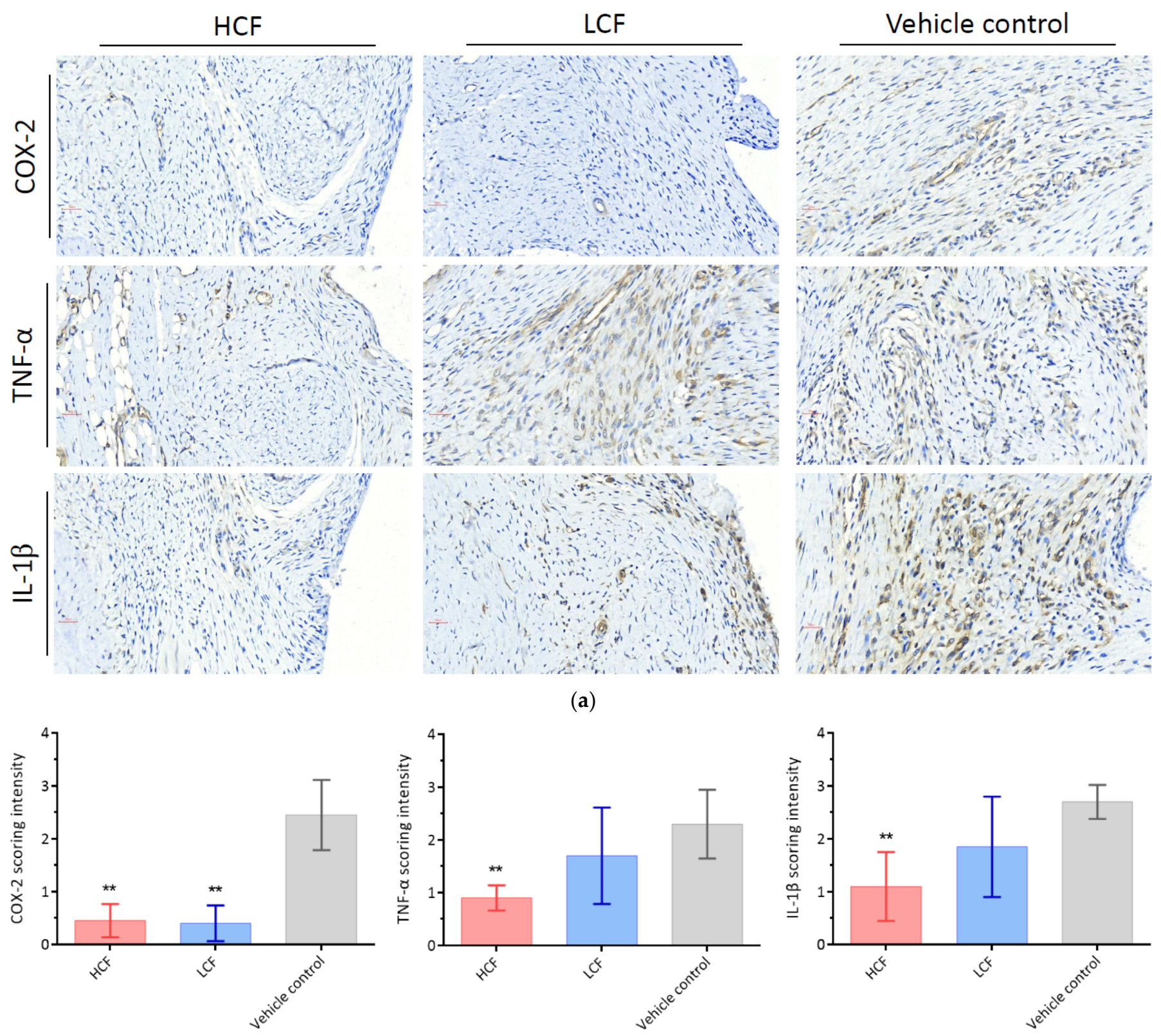

(b)

Figure 4. Immunohistochemical analysis of COX-2, TNF- $\alpha$, and IL-1 $\beta$ expression. (a) Representative histopathological features of the inflammatory status in the infrapatellar fat pad (IFP). (b) Semi-quantification of the COX-2, TNF- $\alpha$, and IL-1 $\beta$ expression scored by the staining intensity within the inflammatory IFP. Scale bar represents $30 \mu \mathrm{m}$. Data are presented as mean \pm S.D. and were analyzed with the Kruskal-Wallis test, followed by Dunn's post hoc test (COX-2, Cyclooxygenase-2; TNF- $\alpha$, tumor necrosis factor-alpha; IL-1 $\beta$, interleukin 1-beta; HCF, high-dose curcumin and FCE p.o.; LCF, low-dose curcumin and FCE p.o.; L, left hindlimb; R, right hindlimb; ${ }^{* *}, p<0.01$ ).

Curcumin and clam extract not only exhibit desirable anti-inflammatory effects in local tissues, but also the systemic inflammatory responses of MIA-induced OA rats significantly decrease. Compared with the VC group, inflammatory factors including MCP-1, IL-1, IL-6, and TNF- $\alpha$ in the serum of rats were significantly reduced when treated with HCF $(p<0.01$, Figure 5). A similar effect on reducing pro-inflammatory cytokines could also be observed in the LCF group, though it only significantly lowered MCP-1 and IL-1 production $(p<0.05$, Figure 5a,b). These results indicate that curcumin and clam extract could have anti-inflammatory effects in the rat OA model. 
(a)

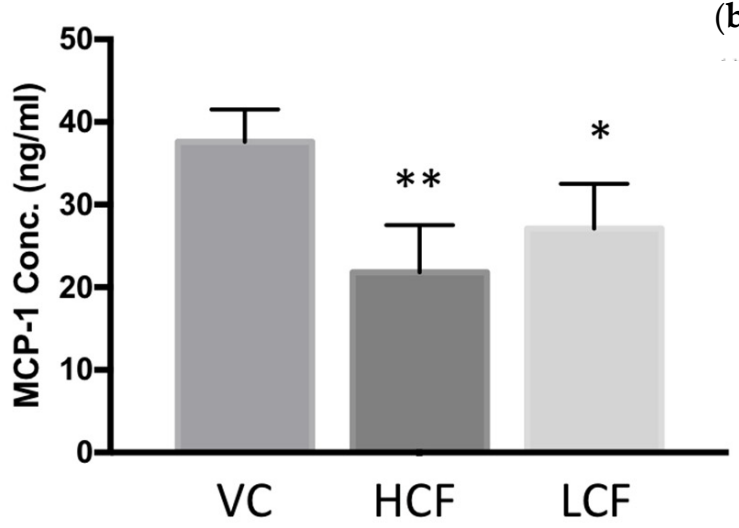

(c)

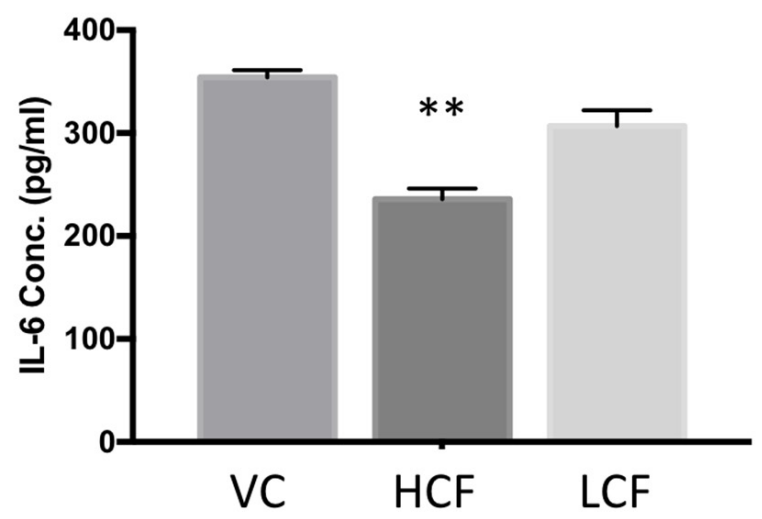

(b)

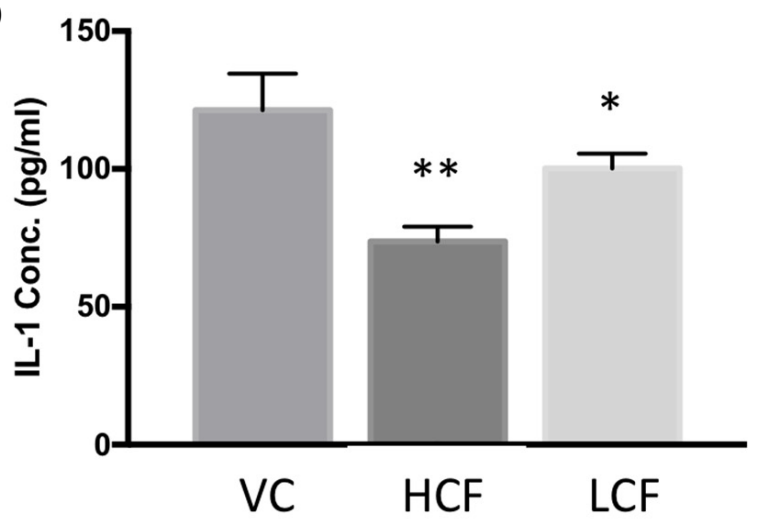

(d)

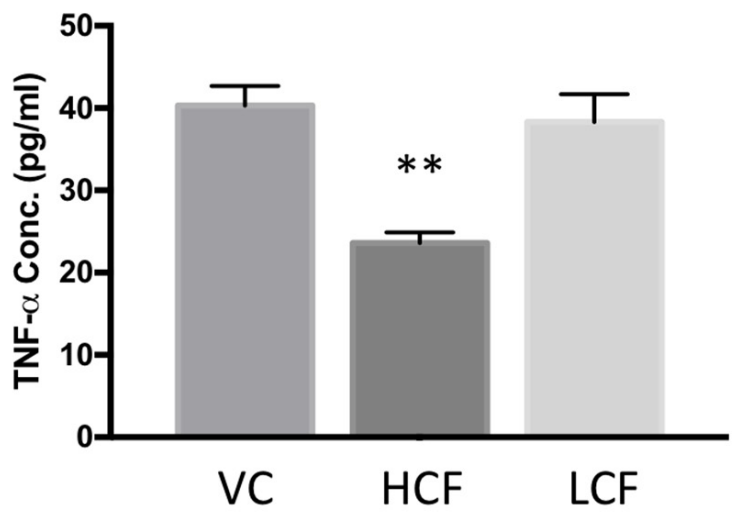

Figure 5. Effect of curcumin and FCE on serum MCP-1 and pro-inflammatory cytokines' levels in MIA-induced rat osteoarthritis. (a) MCP-1, (b) IL-1, (c) IL-6, (d) TNF- $\alpha$. VC: vehicle control; HCF, high-dose curcumin and FCE; LCF, low-dose curcumin and FCE. Data were presented as mean \pm S.D. ${ }^{*} p<0.05$ and ${ }^{* *} p<0.01$ indicate significant differences from the vehicle control group (VC).

\subsection{Curcumin and FCE Can Significantly Promote Cartilage Regeneration in High and Low Doses}

In consideration of standardized and reliable histopathological OA assessment, we employed the OARSI scoring system to fully examine cartilage damage and regeneration in the patellofemoral joint. Briefly, 6 grades (0-6) and 4 stages (0-4) for depth progression of the cartilage and extent of joint involvement were applied as a semi-quantitative method of OA score (score $=$ grade $\times$ stage; range: $0-24$ ). No cartilage degeneration or chondrocyte deaths were observed in the saline-injected right hindlimb. In the left hindlimb from the vehicle control group, however, chondrocyte death, erosion, and severe mineralization of hyaline cartilage were observed (Figure 6a), with an OARSI score of $21.0 \pm 4.24$ (Figure 6b, $p<0.01$ vs. HCF group; $p<0.05$ vs. LCF group). Though mild matrix loss and decrease in cartilage thickness occurred, curcumin and FCE repaired joint damage and promoted cartilage regeneration in OA rats, as indicated by OARSI scores of $4.3 \pm 1.25$ in the HCF group and $4.0 \pm 2.83$ in the LCF group (Figure $6 \mathrm{~b}, p>0.05$ ). These results indicated that the severity of OA significantly decreased, and that cartilage would gradually regenerate with continuous consumption of curcumin and FCE. 

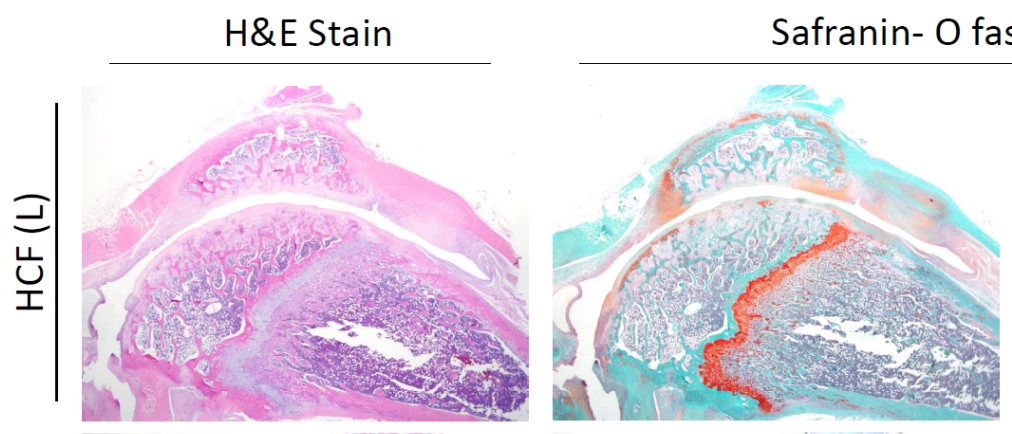

\section{ast green stain}
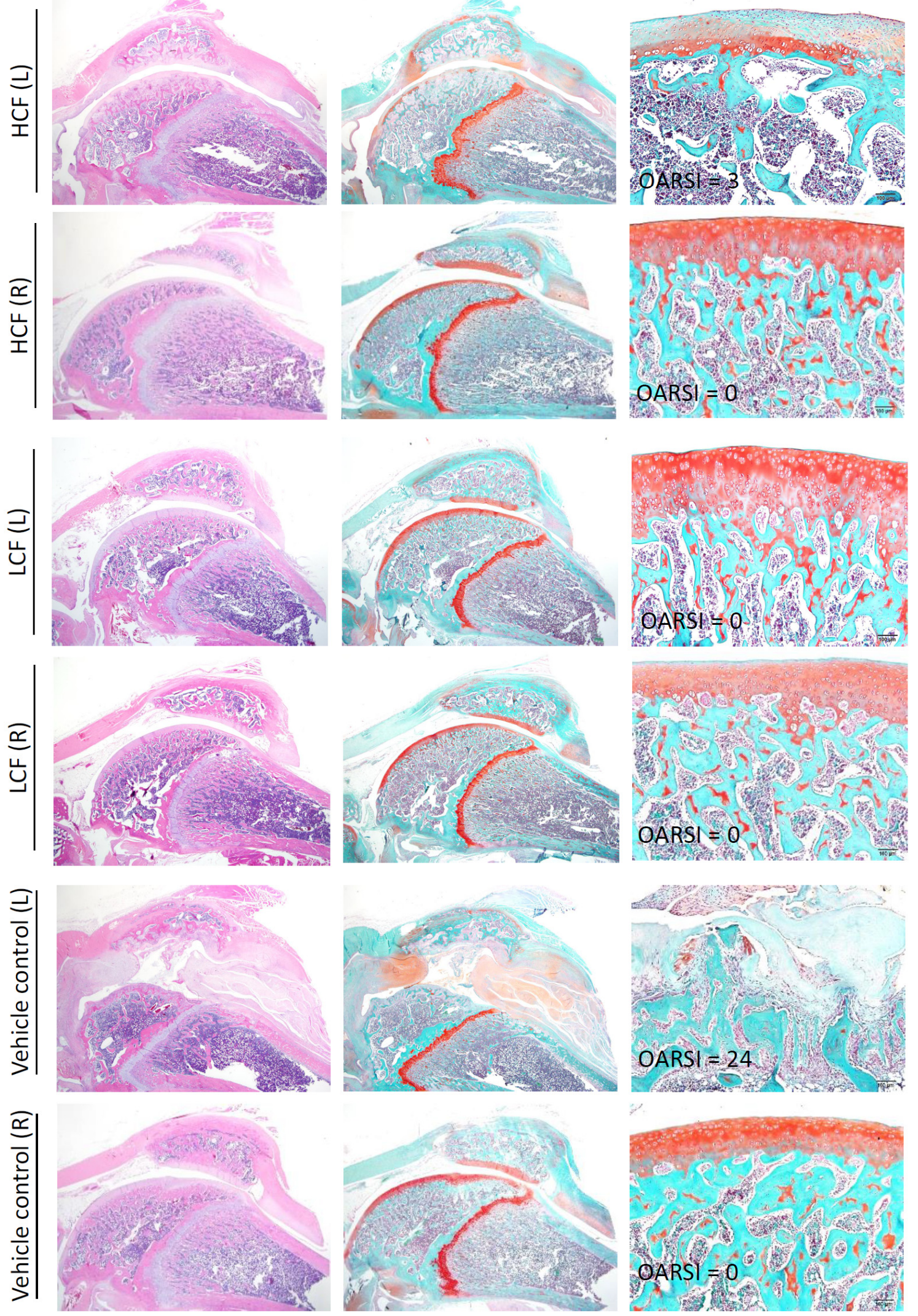

(a)

Figure 6. Cont. 


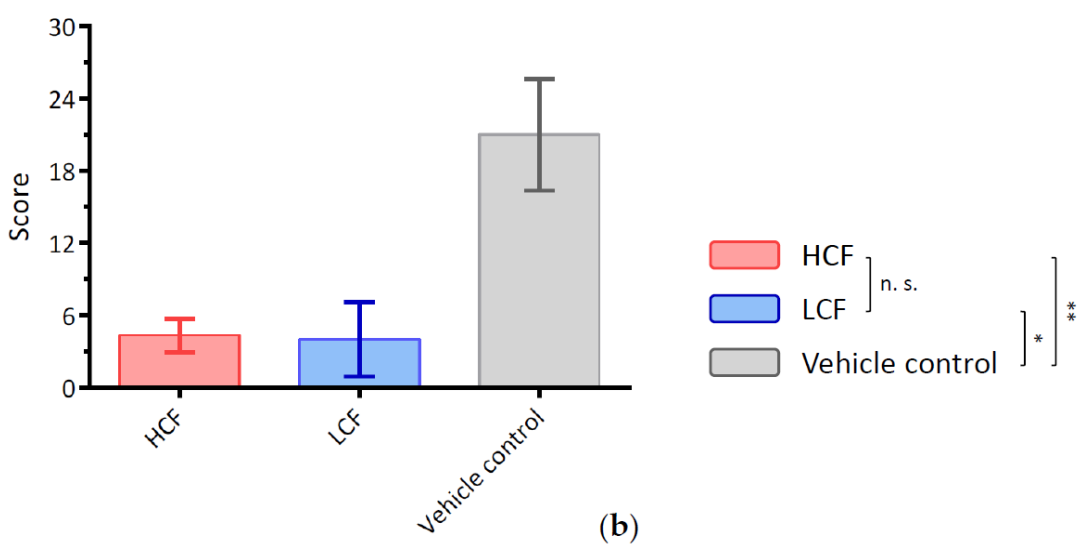

Figure 6. Representative histopathological features of articular cartilage regeneration in patellofemoral joint scored by the Osteoarthritis Research Society International (OARSI) scoring system. (a) Joints were sectioned in the sagittal plane and stained with Hematoxylin and Eosin (H\&E) and safranin-O fast green. Representative articular pathology images in different treatments after MIA or saline administration. Representative OARSI scores are shown in each image. (b) OARSI histological scoring system was employed for evaluation of articular cartilage regeneration. Data are presented as mean \pm S.D. and were analyzed with the Kruskal-Wallis test, followed by Dunn's post hoc test (HCF, high-dose curcumin and FCE p.o.; LCF, low-dose curcumin and FCE p.o.; L, left hindlimb; R, right hindlimb; n.s., no significant difference; ${ }^{*}, p<0.05 ;{ }^{* *}, p<0.01$ ).

\section{Discussion}

Our results demonstrated both clinical improvement and histopathological findings in an MIA-induced OA model. Initially, nociception before and after MIA injection was evaluated in this study. The WBS test was applied, and the upward tendency indirectly implied the clinical progress of MIA-induced OA rats. Additionally, to quantify the severity of IFP inflammation within the joints and OA joint histopathological features, our findings were examined with an IFP inflammation scoring system and OARSI scores.

Evidence appeared to show that the combination of curcumin and FCE had a beneficial effect on OA. Notably, a recovery trend was observed in a dose-dependent manner. Curcumin and FCE can reduce the inflammatory response in the synovium. Microscopically, the fibrotic lesions in the body of the IFP and the thickness of lining cells at the surface of the IFP were significantly improved after daily HCF consumption (Figure 3). Chondrocyte death and mineralization of hyaline cartilage were significantly reduced, and cartilage regeneration was also observed after curcumin and FCE consumption (Figure 6). The regeneration effect may be worthy of attention because NSAIDs fail to slow the progression of OA. In a clinical view, high-dosage curcumin and FCE indeed relieved pain symptoms. After palliation of the severe inflammation in the joints, the rats redistributed their body weight to the MIA-induced limb on day 28 (Figure $1 \mathrm{~b}$ and Table 2). We suggest the possibility of a synergistic effect on alleviating the OA progression and cartilage regeneration due to inhibition of pro-inflammatory cytokines and mediators.

$\mathrm{OA}$ is a whole-joint disease, as the inflammatory environment triggers many immune cells to infiltrate in the tissues [28-31]. Chronic inflammation and cartilage degeneration are two major problems associated with the progression of OA [5]. Chronic inflammation makes clinical signs more severe, and $\mathrm{OA}$ is also characterized by the degradation of articular cartilage and bone matrix [32]. Loss of collagen and the proteoglycan aggrecan leads to the destruction of the cartilage structure and function [4]. Thus, inhibition of aggressive inflammation within joints and reversal of cartilage degradation may largely alleviate the progression of OA patients.

Herein, cytokines and signal pathways related to OA should be briefly described. Interleukin (IL)- $1 \beta$ and TNF- $\alpha$ are considered the most prominent pro-inflammatory cytokines in OA [33,34]. These two cytokines suppress the expression of type II collagen, cleave the articular cartilage matrix, and release proteolytic enzymes to destroy the cartilage [34-36]. 
Activation of an inflammatory signal pathway, NF- $\kappa \mathrm{B}$, leads to the upregulation of COX-2, IL-1 $\beta$, and TNF- $\alpha$ and a chronic inflammatory state within the joints [33,37].

Physiological microenvironments can be modulated or influenced by curcumin and FCE. Curcumin can inhibit the production of inflammatory mediators such as nitric oxide (NO), prostaglandin2 (PGE2), IL-6, and IL-8 stimulated by IL-1 $\beta$ [11,12,38]. Additionally, curcumin prevents IL- $1 \beta$-induced NF- $\mathrm{KB}$ activation and translocation, giving rise to reduced expression of the NF- $\mathrm{kB}$ downstream pro-inflammatory gene COX-2 [39]. Through inhibition of the NF- $\mathrm{KB}$ pathway, the expression of inflammatory OA targets can be reduced. These targets are IL- $1 \beta$, TNF- $\alpha$, COX-2, and matrix metalloproteinases (MMPs). MMPs, especially MMP-13, are the major factors in inflammation and extracellular matrix degradation in OA progression [11]. Furthermore, curcumin exerts upstream control over NF- $\mathrm{kB}$, inhibiting the pathway of toll-like receptor 4 (TLR4), which mediates the inflammatory responses involved in OA pathogenesis [40]. Inhibition of NF- $\mathrm{kB}$ also displays a protective function from the accumulation of advanced glycation end-products (AGEs) and upregulation of MMP-13, which can retard OA progression by reducing inflammation and cartilage ageing [41]. FCE has a similar property of inhibitory effects of NF-kB, but it also downregulates mitogen-activated protein kinases (MAPKs), resulting in declines in NO, IL-1 $\beta$, IL-6, and TNF- $\alpha$ as well [42].

In summary, the present study illustrated the synergistic effects of curcumin and FCE on alleviating the progression of OA by anti-inflammatory function and cartilage regeneration. Curcumin downregulates the activity of COX-2 [18] and targets the NF- $\mathrm{kB}$ pathway to inhibit the release of pro-inflammatory cytokine TNF- $\alpha$ [19]. FCE neutralizes inflammation and slows the destruction of articular cartilage by reducing IL-1 $\beta$ and TNF$\alpha$ [23]. The anti-inflammatory function delays the progression of ageing or mineralization of cartilage, thereby allowing the articular cartilage to regenerate. Pain symptoms are ultimately eased by the inhibition of inflammation and regeneration of cartilage. The results suggest that curcumin and FCE have the potential to be an alternative remedy in the management of $\mathrm{OA}$ or other inflammatory diseases.

Author Contributions: Conceptualization, C.-S.L. and Y.-L.L.; methodology, C.-S.L.; validation, C.-H.H. and W.-H.H.; formal analysis, C.-H.K.; investigation, H.-P.W. and Y.-T.K.; data curation, Y.L.L.; writing—original draft preparation, C.-H.K.; writing—review and editing, C.-S.L.; supervision, C.-S.L.; project administration, C.-H.H. and Y.-T.K.; funding acquisition, C.-S.L. All authors have read and agreed to the published version of the manuscript.

Funding: This research was funded by Council of Agriculture, Taiwan, grant numbers 109AS-17.1.7AD-U1 and 110AS-10.1.7-AD-U1. The APC was funded by 110AS-10.1.7-AD-U1.

Institutional Review Board Statement: The study was conducted according to the guidelines of the Declaration of Helsinki, and approved by the Institutional Animal Care and Use Committee of National Taiwan University, Taipei, Taiwan (protocol code: IACUC No. NTU107-EL-00189, 6 March 2019).

Data Availability Statement: The data presented in this study are available on request from the corresponding author.

Conflicts of Interest: The authors declare no conflict of interest.

\section{References}

1. Kuyinu, E.L.; Narayanan, G.; Nair, L.S.; Laurencin, C.T. Animal models of osteoarthritis: Classification, update, and measurement of outcomes. J. Orthop. Surg. Res. 2016, 11, 19. [CrossRef]

2. Yu, S.P.; Hunter, D.J. Managing osteoarthritis. Aust. Prescr. 2015, 38, 115-119. [CrossRef]

3. Udo, M.; Muneta, T.; Tsuji, K.; Ozeki, N.; Nakagawa, Y.; Ohara, T.; Saito, R.; Yanagisawa, K.; Koga, H.; Sekiya, I. Monoiodoacetic acid induces arthritis and synovitis in rats in a dose- and time-dependent manner: Proposed model-specific scoring systems. Osteoarthr. Cartil. 2016, 24, 1284-1291. [CrossRef]

4. Grässel, S.; Muschter, D. Recent advances in the treatment of osteoarthritis. F1000Research 2020, 9. [CrossRef] [PubMed]

5. Ma, Y.; Guo, H.; Bai, F.; Zhang, M.; Yang, L.; Deng, J.; Xiong, L. A rat model of knee osteoarthritis suitable for electroacupuncture study. Exp. Anim. 2018, 67, 271-280. [CrossRef] [PubMed] 
6. Bijlsma, J.W.; Berenbaum, F.; Lafeber, F.P. Osteoarthritis: An update with relevance for clinical practice. Lancet 2011, 377, 2115-2126. [CrossRef]

7. Losina, E.; Katz, J.N. Total joint replacement outcomes in patients with concomitant comorbidities: A glass half empty or half full? Arthritis Rheum. 2013, 65, 1157. [CrossRef] [PubMed]

8. $\quad$ Legg, D.O.; Reda, D.J.; Harris, C.L.; Klein, M.A.; O’Dell, J.R.; Hooper, M.M.; Bradley, J.D.; Bingham III., C.O.; Weisman, M.H.; Jackson, C.G.; et al. Glucosamine, chondroitin sulfate, and the two in combination for painful knee osteoarthritis. N. Engl. J. Med. 2006, 354, 795-808. [CrossRef]

9. Savvidou, O.; Milonaki, M.; Goumenos, S.; Flevas, D.; Papagelopoulos, P.; Moutsatsou, P. Glucocorticoid signaling and osteoarthritis. Mol. Cell Endocrinol. 2019, 480, 153-166. [CrossRef] [PubMed]

10. McAlindon, T.E.; Bannuru, R.R.; Sullivan, M.C.; Arden, N.K.; Berenbaum, F.; Bierma-Zeinstra, S.M.; Hawker, G.A.; Henrotin, Y.; Hunter, D.J.; Kawaguchi, H.; et al. OARSI guidelines for the non-surgical management of knee osteoarthritis. Osteoarthr. Cartil. 2014, 22, 363-388. [CrossRef]

11. D'Adamo, S.; Cetrullo, S.; Panichi, V.; Mariani, E.; Flamigni, F.; Borzì, R.M. Nutraceutical Activity in Osteoarthritis Biology: A Focus on the Nutrigenomic Role. Cells 2020, 9, 1232. [CrossRef]

12. Cheragh-Birjandi, S.; Moghbeli, M.; Haghighi, F.; Safdari, M.R.; Baghernezhad, M.; Akhavan, A.; Ganji, R. Impact of resistance exercises and nano-curcumin on synovial levels of collagenase and nitric oxide in women with knee osteoarthritis. Transl. Med. Commun. 2020, 5, 3. [CrossRef]

13. Green, J.A.; Hirst-Jones, K.L.; Davidson, R.K.; Jupp, O.; Bao, Y.; MacGregor, A.J.; Donell, S.T.; Cassidy, A.; Clark, I.M. The potential for dietary factors to prevent or treat osteoarthritis. Proc. Nutr. Soc. 2014, 73, 278-288. [CrossRef]

14. Kuo, I.M.; Lee, J.J.; Wang, Y.S.; Chiang, H.C.; Huang, C.C.; Hsieh, P.J.; Han, W.; Ke, C.H.; Liao, A.T.C.; Lin, C.S. Potential enhancement of host immunity and anti-tumor efficacy of nanoscale curcumin and resveratrol in colorectal cancers by modulated electro- hyperthermia. BMC Cancer 2020, 20, 603. [CrossRef] [PubMed]

15. Keshavarz, R.; Bakhshinejad, B.; Babashah, S.; Baghi, N.; Sadeghizadeh, M. Dendrosomal nanocurcumin and p53 overexpression synergistically trigger apoptosis in glioblastoma cells. Iran. J. Basic Med. Sci. 2016, 19, 1353-1362. [CrossRef] [PubMed]

16. Jurenka, J.S. Anti-inflammatory properties of curcumin, a major constituent of Curcuma longa: A review of preclinical and clinical research. Altern. Med. Rev. 2009, 14, 141-153.

17. Fadus, M.C.; Lau, C.; Bikhchandani, J.; Lynch, H.T. Curcumin: An age-old anti-inflammatory and anti-neoplastic agent. J. Tradit. Complementary Med. 2017, 7, 339-346. [CrossRef] [PubMed]

18. Zhang, F.; Altorki, N.K.; Mestre, J.R.; Subbaramaiah, K.; Dannenberg, A.J. Curcumin inhibits cyclooxygenase-2 transcription in bile acid- and phorbol ester-treated human gastrointestinal epithelial cells. Carcinogenesis 1999, 20, 445-451. [CrossRef] [PubMed]

19. Liu, Z.; Ying, Y. The Inhibitory Effect of Curcumin on Virus-Induced Cytokine Storm and Its Potential Use in the Associated Severe Pneumonia. Front. Cell Dev. Biol. 2020, 8, 479. [CrossRef]

20. Huang, K.-C.; Wu, W.-T.; Yang, F.-L.; Chiu, Y.-H.; Peng, T.-C.; Hsu, B.-G.; Liao, K.-W.; Lee, R.-P. Effects of freshwater clam extract supplementation on time to exhaustion, muscle damage, pro/anti-inflammatory cytokines, and liver injury in rats after exhaustive exercise. Molecules 2013, 18, 3825-3838. [CrossRef] [PubMed]

21. Peng, T.C.; Subeq, Y.M.; Lee, C.J.; Lee, C.C.; Tsai, C.J.; Chang, F.M.; Lee, R.P. Freshwater clam extract ameliorates acute liver injury induced by hemorrhage in rats. Am. J. Chin. Med. 2008, 36, 1121-1133. [CrossRef] [PubMed]

22. Hsu, C.L.; Hsu, C.C.; Yen, G.C. Hepatoprotection by freshwater clam extract against CCl4-induced hepatic damage in rats. Am. J. Chin. Med. 2010, 38, 881-894. [CrossRef] [PubMed]

23. Lin, C.M.; Lin, Y.L.; Tsai, N.M.; Wu, H.Y.; Ho, S.Y.; Chen, C.H.; Liu, Y.K.; Chiu, Y.H.; Ho, L.P.; Lee, R.P.; et al. Inhibitory effects of chloroform extracts derived from Corbicula fluminea on the release of pro-inflammatory cytokines. J. Agric. Food Chem. 2012, 60, 4076-4082. [CrossRef] [PubMed]

24. Sim, B.Y.; Choi, H.J.; Kim, M.G.; Jeong, D.G.; Lee, D.G.; Yoon, J.M.; Kang, D.J.; Park, S.; Ji, J.G.; Joo, I.H.; et al. Effects of ID-CBT5101 in Preventing and Alleviating Osteoarthritis Symptoms in a Monosodium Iodoacetate-Induced Rat Model. J. Microbiol. Biotechnol. 2018, 28, 1199-1208. [CrossRef] [PubMed]

25. Takahashi, I.; Matsuzaki, T.; Kuroki, H.; Hoso, M. Induction of osteoarthritis by injecting monosodium iodoacetate into the patellofemoral joint of an experimental rat model. PLoS ONE 2018, 13, e0196625. [CrossRef]

26. Pritzker, K.P.; Gay, S.; Jimenez, S.A.; Ostergaard, K.; Pelletier, J.P.; Revell, P.A.; Salter, D.; van den Berg, W.B. Osteoarthritis cartilage histopathology: Grading and staging. Osteoarthr. Cartil. 2006, 14, 13-29. [CrossRef]

27. Grevenstein, D.; Heilig, J.; Dargel, J.; Oppermann, J.; Eysel, P.; Brochhausen, C.; Niehoff, A. COMP in the Infrapatellar Fat Pad-Results of a Prospective Histological, Immunohistological, and Biochemical Case-Control Study. J. Orthop. Res. 2020, 38, 747-758. [CrossRef]

28. Goldring, M.B.; Otero, M. Inflammation in osteoarthritis. Curr. Opin. Rheumatol. 2011, 23, 471-478. [CrossRef]

29. He, Q.; Sun, C.; Lei, W.; Ma, J. SOCS1 Regulates Apoptosis and Inflammation by Inhibiting IL-4 Signaling in IL-1 $\beta$-Stimulated Human Osteoarthritic Chondrocytes. BioMed Res. Int. 2017, 2017, 4601959. [CrossRef]

30. Bian, Y.; Zhang, M.; Wang, K. Taurine protects against knee osteoarthritis development in experimental rat models. Knee 2018, 25, 374-380. [CrossRef]

31. Jenei-Lanzl, Z.; Meurer, A.; Zaucke, F. Interleukin-1ß signaling in osteoarthritis-chondrocytes in focus. Cell Signal 2019, 53, 212-223. [CrossRef] [PubMed] 
32. Goldring, M.B.; Marcu, K.B. Cartilage homeostasis in health and rheumatic diseases. Arthritis Res. Ther. 2009, 11, 224. [CrossRef] [PubMed]

33. Chung, H.Y.; Lee, E.K.; Choi, Y.J.; Kim, J.M.; Kim, D.H.; Zou, Y.; Kim, C.H.; Lee, J.; Kim, H.S.; Kim, N.D.; et al. Molecular Inflammation as an Underlying Mechanism of the Aging Process and Age-related Diseases. J. Dent. Res. 2011, 90, 830-840. [CrossRef] [PubMed]

34. Leong, D.J.; Choudhury, M.; Hirsh, D.M.; Hardin, J.A.; Cobelli, N.J.; Sun, H.B. Nutraceuticals: Potential for chondroprotection and molecular targeting of osteoarthritis. Int. J. Mol. Sci. 2013, 14, 23063-23085. [CrossRef]

35. Kapoor, M.; Martel-Pelletier, J.; Lajeunesse, D.; Pelletier, J.P.; Fahmi, H. Role of proinflammatory cytokines in the pathophysiology of osteoarthritis. Nat. Rev. Rheumatol. 2011, 7, 33-42. [CrossRef] [PubMed]

36. Henrotin, Y.; Deby-Dupont, G.; Deby, C.; De Bruyn, M.; Lamy, M.; Franchimont, P. Production of active oxygen species by isolated human chondrocytes. Br. J. Rheumatol. 1993, 32, 562-567. [CrossRef]

37. Millerand, M.; Berenbaum, F.; Jacques, C. Danger signals and inflammaging in osteoarthritis. Clin. Exp. Rheumatol. 2019, 37, 48-56. [PubMed]

38. Mathy-Hartert, M.; Jacquemond-Collet, I.; Priem, F.; Sanchez, C.; Lambert, C.; Henrotin, Y. Curcumin inhibits pro-inflammatory mediators and metalloproteinase-3 production by chondrocytes. Inflamm. Res. 2009, 58, 899-908. [CrossRef]

39. Csaki, C.; Mobasheri, A.; Shakibaei, M. Synergistic chondroprotective effects of curcumin and resveratrol in human articular chondrocytes: Inhibition of IL-1beta-induced NF-kappaB-mediated inflammation and apoptosis. Arthritis Res. Ther. 2009, 11, R165. [CrossRef]

40. Yan, D.; He, B.; Guo, J.; Li, S.; Wang, J. Involvement of TLR4 in the protective effect of intra-articular administration of curcumin on rat experimental osteoarthritis. Acta Cir. Bras. 2019, 34. [CrossRef] [PubMed]

41. Yang, Q.; Wu, S.; Mao, X.; Wang, W.; Tai, H. Inhibition effect of curcumin on TNF- $\alpha$ and MMP-13 expression induced by advanced glycation end products in chondrocytes. Pharmacology 2013, 91, 77-85. [CrossRef] [PubMed]

42. Hua, K.F.; Chen, G.M.; Ho, C.L.; Chen, M.C.; Chen, Y.L.; Chen, W.J.; Huang, J.F.; Perng, Y.S.; Lin, C.C. Freshwater Clam Extract Inhibits Inflammatory Responses in LPS-Activated Macrophages by Reducing the Activation of Mitogen-Activated Protein Kinases and NF-кB. Nat. Prod. Commun. 2012, 7, 1435-1440. [CrossRef] [PubMed] 